\section{THE ACTION OF EXPECTORANTS.*}

BY

J. A. GUNN, M.A., M.D., D.Sc., Professor of Pharmacology in the University of Oxford, President of the Section.

Whes we take a broad survey of the field of therapeuties it would be fair to say that, though from one year to another there may be no dramatic advance, yet in the course of a decade or so, especially during the last halfcentury, there is hardly any branch of therapeutics which fails to show steady progress. This may be either in the way of discovery of new remedies, or in the more full and precise knowledge of the action and uses of older ones. When we think, for example, of such important groups as local and general anaesthetics, hypnotics, antiseptics, and siecific remedies, instances of real advance in a few years spring to the mind. But there is one department of therapeutics in which little forward movement has been registered, not only in a decade, but hardly any even in the last half-century, and that is the group of expectorants. Indeed, I am not convinced that we are even so skilled in the use of expectorants as our grandfathers were, for, with less to learn, they had more time-and I sometimes think more enthusiasm - for observing at the bedside the action of drugs.

It may be profitable first of all to consider the reasons for this peculiar position in which the expectorants are placed. The first reason is the difficulty of measurement. When an effect can easily be measured, then advance is likely to be rapid. If it were as easy to determine the quantity of bronchial secretion as it is to measure the amount of urine, there would be by this time no such vagueness in regard to the effect of expectorants as still exists. In a case of bronchitis the amount of sputum may vary from time to time. It is difficult to get a control, and it is impossible to separate the saliva from the bronchial discharge. But in spite of these difficulties I venture to think that something more might be attempted in the way of actual measurement of the amount of sputum, so as to dispel the present confusion as to whether a substance increases or diminishes the bronchial secretion, or to discover in what clinical conditions an increase is certainly brought about, and how long it lasts. The chief clinical difficulty, however, is the absence of quantitative measurement.

But often where the clinical difficulties are great the problem can be solved by experiments on normal men or on lower animals. Here again, however, there are unusual obstacles in the way of estimating the effects of expectorants. For example, a distinguished physiologist, who had taken large quantities of ammonium chloride, mentioned to me that he had not observed any expectorant action from it; this is, perhaps, not so difficult to explain even if ammonium chloride has an expectorant action in disease. Normally we are quite unconscious of the existence of our bronchi. There is just sufficient secretion of the mucous surface to keep the passages moist; any excess of secretion is carried up by the cilia and swallowed. A healthy person need not cough; so that unless the secretion is so copious as to require coughing up-that is to say, is interfering with the channel-it is doubtful whether an increase of secretion would be brought to consciousness. On the other hand, in a patient with an inflamed bronchial mucosa with viscid sputum producing cough, a slight increase in the amount of secretion with lessened viscidity might enable the bronchi to be cleared at least tomporarily, and the effect of an expectorant which would produce no noticeable effect on a normal person may be immediately discernible and grateful to a patient in such a condition. Normal people are therefore not good subjects for testing the action of expectorants. In laboratory experiments some of the difficulties met with in clinical observations can be overcome, but new ones a:e introduced. Measurement of the amount of bronchial secretion is easier. Such methods can be employed, for example, as applying small measured and weighed pieces

* A paper read before the Section of Therapeutics and Pharmacology at the Anuual Meeting of the British Medical Association, July, 1927. of absorbent paper to the trachea and comparing the relative increase in weight before and after the administration of expectorants. But other factors have to be reckoned with-for example, differences in reaction between a normal and an inflamed bronchus, the complicating effects of anaesthetics; and the differences between various species of animals. In brief, there are difficulties in the way of exact estimation of the action of expectorants, whether the experiments be done on normal or diseased persons or on laboratory animals.

Even the definition of expectorant is unsatisfactory. From its derivation the word originally meant something that aids the expulsion of a discharge from the chest, but it has come to be applied loosely to substances which either increase or decrease the discharge, and the word has also special associations with the treatment of cough.

The Physiological Basis of Expectorant action.

In order to understand the action of expectorants it is necessary to appreciate what is the physiological mechanism for protecting the air passages. As is usual with mucous mepubranes there is a double mechanism for this-a motor and a secretory. In the case of the thoracic air passages, with which only we are immediately concerned, there is an elaborate and highly ingenious motor mechanism; there are, in fact, three mechanisms. First, there is the propulsive movement of the cilia which line the mucous membranes; secondly, there is the co-ordinated reflex expulsive mechanism of cough; and lastly, there is the peristaltic movement of the muscle of the smaller bronchi. Now each of these motor mechanisms has its definite part to play in the scheme; the failure of one renders the whole mechanism defective, though not inoperative, because their activities overlap. They perform somewhat different functions; and are called into play under different conditions.

Let us take first the patient, continual movement of tho cilia. The trachea and bronchi aro lined with ciliated epithelium down to, but excluding, a short length of the terminal bronchioles. I remember, as a medical student, thinking that the propulsive action of microscopic cilia was one of those mythical forces that operate only in textbooks. It required only a slight experience of ciliary movement to alter this opinion. A class demonstration by Professor Schafer, in which was shown, projected by lantern, a piece of cork swept on by the ciliary movement of a strip of frog's gullet was an awakening and convincing demonstration of the power of ciliary movement. If properly aligned, a small strip of ciliated epithelium can be made to rotate a revolving drum. The ciliary movement is, in fact, a powerful propulsive movement, and it is this motor mechanism alone which normally deals with removal of bronchial secretion; cough is an exceptional and reserve mechanism. Continual secretion of mucus is necessary to keep the air passages moist, but with normal variations in this secretion the ciliary movement is competent to deal. It acts like a noving staircase, continually carrying up any excessivi secretion till it reaches the throat and is swallowed. It has been calculated that this movement may propel solid particles at the rato of ono inch a minute. The speed will vary with the sizo of the particles and witl the viscidity of the secretion, l,ut, ninder favourable conditions, the ciliary movement is able unaided to bring up material from the very depths of the bronchioles in a few minutes.

A description of the expulsive mechanism of cough is unnecessary here. One point, lowerer, which it is important to bear in mind is that the expulsive effect of cough is greatest nearer tho larynx, and gets less nearer tho lungs. Indeed, it has been stated that the expulsive force of cough affects the smaller bronchioles very slightly, if at ail. It will be seen, therefore, that the smaller branches of the bronchioles, those which enter the lung alveoli, are in a parlous condition-they have no cilia; they are not reached by cough. It is here that the third motor mechanism comes into play. A definite peristaltic movement of bronchial muscle, as contrasted with a mere alteration in tone of this muscle, is supposed to be a property especially present in the muscle of the finer bronchi. Where cough and cilia fail this peristaltic movement takes their 
place. If this be so there is here revealed, as in all the regulating processes of the body, an extraordinarily ingenious and concerted mechanism.

In regard to the second protective mechanism-the bronchial secretion-this is necessary to keep the bronchial surface moist and to dilute irritants, and for this purpose the bronchial mucous membrane is supplied with a great wealth of glands. We seem to have little knowledge as to the regulation of this secretion under normal conditionsas to whether, for example, these glands are capable of altering the consistency or the composition of the secretion, or whether this remains uniform. If the latter, then the consistency will subsequently vary, for example, according to the speed of evaporation of water, and consequently with the dryness of the air or the quickness of respiration. It may be conjectured that if this cvaporation be excessivo and an undue viscidity of the mucus result, this viscidity will irritate the mucous membrane and cause a refflexly increased secretion. So could the consistency of the secretion be regulated.

This motor and secretory mechanism is in great part regulated by the nervous system. Afferent fibres of the vagus pass centrally from the mucous membrane; efferent fibres of the vagus pass down to the bronchial muscle and $t_{0}$ the secretory glands. The bronchial muscle is also supplied by efferent fibres of the sympathetic. These afferent and efferent fibres converge upon a somewhat hypothetical cough centre which is related to, if not actually an integral part of, the respiratory and vomiting centres. The cilia are apparently not under direct nervous influence.

It is beyond the scope of this paper to enter fully into this nervous control. It will suffice for the present purpose to recall that under physiological conditions the movement of the cilia together with a continual and regular secretion of the glands is necessary for maintaining the integrity of the bronchial mucous membrane. Under abnormal conditions, when the mucous membrane is irritated by noxious particles or by too profuse or too viscid secretion, this irritation stimulates the cough centro and there results reflexly either cough, or increased secretion, or peristaltic movement of the bronchioles, or some combination of these. It is in consonance with the view that the expulsive effect of cough is effective mainly in the upper air passages that it is elicited also most easily by irritation of the nucous nembrano of this area.

\section{Method of Action of Expectorants.}

I propose to deal almost exclusively with the methods by which the bronchial secretion can be increased by drugs, especially with a view to suggesting reasons for some clinical differences in the action of expectorants, of which no explanation seems to have been given. Expectorants may increase the bronchial secretion by acting in one, or a combination, of four different ways, depending upon their site of action. Upon these differences can be based a physiological classification of them. They may act (1) reflexly; (2) by stimulation of the vagus centre; (3) by stimulation of the secretory (vagal) terminations; (4) ky direct stimulation of the secretory glands. This classification is not to be regarded as a mere academic curiosity. It is upon differences in site of action that the superior suitability of certain expectorants for certain conditions largely depends.

\section{Reflex Expectorants.}

A large number of drugs which have no common pharmacological property, other than that of being gastric irritants, have in the course of time come to be used empirically as secretory expectorants when given by the mouth. Many of these drugs have been used as emetics in larger doses. That there is some association between an emetic and an expectorant action has been known for a century or more. So far as I can find, Christison was the first to give something of an explanation of this when he stated (1842), in regard to ipecacuanha, that " the pulmonary mucous membrane would seem to be often stimulated to increased secretion by repeated small doses, although nausea be not produced, probably in consequence of the lungs sympathizing with a gentle stimulus and increased secretion of the gastric mucous membrane."
A more modern explanation of this is that the increased secretion of the bronchi produced by ipecacuanha and other members of this group is a reflex, the afferent stimulus for which is the irritation of the gastric mucous membrane; the afferent path the sensory fibres of the vagus; the centre the romiting centre (or possibly a centre for bronchial secretion); and the efferent path the secretory fibres of the vagus going to the bronchial glands.

What is a little unusual about this reflex is that normally the afferent stimulus for reflex bronchial secretion is irritation, not of the gastric, but of the bronchial, mucous membrane. But it is a characteristic feature of vagal reflexes generally that the efferent side of a reflex can be discharged by stimulation of afferent branches of the vagus other than those normally concerned with the reflex, provided that this abnormal stimulation is of sufficient intensity and duration. This, as is well known, is equally true, for example, of the cough reflex. Cough can be induced not only by the normal stimulus, which is irritation of the tracheal or bronchial mucous membrane, but by irritation of other afferent branches of the vagus-as, for example, in the ear, pharynx, or pleura, provided that it is of sufficient intensity and duration. A large number of expectorants belong to this group: ipecacuanha, tartar emetic, ammonium carbonate, squill, and senega all owe their expectorant action, at least in part, to gastric irritation.

I wish to consider, in regard to this type of expectorant action, certain points to which $I$ have seen little or no, reference made. It is well known that reflex bronchial secretion can be induced by any of these gastric irritants with doses which are insufficient to cause actual vomiting, but only certain emetics have come to be used as expectorants; for example, clinical experience has decided in favour of tartar emetic and ipecacuanha in preference to copper sulphate or zinc sulphate. This preference is not accidental. A reflex emetic must possess certain qualities if it is to be suitable for use as an expectorant, and those qualities can be illustrated by a comparison. Copper and zinc sulphate irritate the mucous membrane of the stomach by combining with the proteins of the mucous membrane of the stomach. It is a rapid chemical action, giving rise to a sudden and rather intense stimulation of the afferent nerve ends; emesis, if it is to occur at all, takes place rapidly. These emetics cannot be administered repeatedly, as their chemical action would thereby cause damage to the gastric mucous membrane. The action of tartar emetic and ipecacuanha, on the other hand, is much slower but lasts longer. When, for example, they are used as emetics, the onset of emesis may bo delayed for fifteen to thirty minutes, during which time sensations of gastric irritation are experienced, and vomiting is apt to be repeated even with a single dose. Moreover, when repeated small doses are given no serious gastric irritation is produced. It is fairly obvious, therefore, that there are two qualities which an emetic must possess before it can be of practical value, in smaller doses, as an expectorant. In the first place, it must not produce so gross or intense an irritation of the gastric mucous membrane as to cause serious or permanent gastritis when given repeatedly. In the second place, this milder gastric irritation must be sufficiently prolonged to cause a reflex bronchial secretion of sufficient duration to make the administration of the substance for this purpose worth while.

There is another point in regard to expectorants of this type for which some explanation must be found. The statement that ipecacuanha is a more effective and more certain expeciorant in children than in adults has been made so often by different observers that it must have some basis in fact. The pertinent fact may be that the cmetic centre in children is more active and more sensitive than in adults. It is plausible to suppose that the reflex effects which accompany gastric irritation, of which the exf.ectorant action is one, will also be more easily elicited in children.

The opinion has been widely held that ipecacuanha and tartar emetic have, compared with potassium iodide, some superior merit in acute bronchitis. A reasonable explanation for this would be that the former have a diaphoretic 
action, which would be of value in lowering the temperature in febrile cases. This added effect is not produced by potassium iodide to the same extent.

\section{Central Expectorants.}

Bronchial secretion can be excited not only reflexly but also by stimulation of the centre in the medulla. As has been already pointed out, this centre is closely associated with the vomiting centre, and therefore emetics which act centrally can be used, in subemetic doses, also as expectorants. Ipecacuanha probably acts to some extent centrally, and possibly also tartar emetic, though they act mainly by gastric irritation. Apomorphine, however, acts entirely by central stimulation, and it has been used to some extent as an expectorant. It has not proved therapeutically so valuable as ipecacuanha, probably because its action is too transient.

\section{Stimulants of Secretory Nerve-ends.}

A number of drugs are known which stimulate the terminations of parasympathetic nerves, and consequently stimulate bronchial secretion, among a variety of other effects. The only one of this group which has been used therapeutically is jaborandi, or its active principle pilocarpine. The employment of pilocarpine for this purpose has been almost given up. Great accuracy of dosage is required in prescribing it, otherwise so many unwanted effects, such as constriction of the bronchi, oedema of the lung, profuse sweating, and cardiac slowing, occur coincidently with increased bronchial secretion.

It is possible, however, that there mav still be a limited field of use for pilocarpine if the necessary accuracy of dosage could be attained. It has been observed that, especially in children, the bronchial secretion is increased by pilocarpine before other secretions, but, seeing that there are so many safer expectorants, this fact, even if advantage could be taken of it, would hardly outweigh the disadvantages of pilocarpine, unless possibly in one condition. When the central nervous system is profoundly depressed it is possible that reflex emeties like ipecacuanha are relatively inactive, whereas the action of pilocarpine, which is independent of the condition of the central nervous srstem, would be unaffected. In these circunistances pilocarpine might be the most reliable expectorant.

\section{Direct Stimulants of the Bronchial Glands.}

It was at one time thought that nearly all expectorants produce their effects by being excreted through the bronchial glands and increasing their secretion during passage through them. The action of the majority of expectorants has now been otherwise explained, but certain expectorant salts act in this way. The most importaut of these is potassium iodide. Iodides, like chlorides, are excreted by the bronchial among other glands; but the action of iodides on secretions is not simply due to salt action, as is shown by the fact that in iodism there is a pronounced swelling and increased secretory activity of the nasal, lacrymal, and bronchial glands such as does not occur with chlorides.

The action of iodides, like that of pilocarpine, on the bronchial secretion is independent of the condition of the central nervous system, and they are very reliable expectorants. There are two points in regard to the clinical case of iodides for which no entirely satisfactory explanation has been given. In the first place, the opinion is widely held that iodiles are less useful expectorants for children than for adults. A partial explanation for this may be that iodides lack the diaphoretic and antipretic action of the ipecacuanha group, an action which is of value in febrile bronchitis in children. Secondly, iodides are believed to have a peculiar value in some forms of chronic bronchitis. It is possible that this may be due, though exact measurements are wanting, to a more prolonged action than ipecacuanha, for example. It is also possible that in chronic inflammation of the bronchi iodides may have some action in resolving inflammatory deposits, similar to the known action of iodides in resolving gummatous material.
Other Chassifications of Expectorants.

Classifications of expectorants other than that depending on their site of action have been used. There serms to be no known pharmacological justification for the attempt to divide them into stimulant and depressant expectorants. For example, senega and squill are classed with the former, and ipecacuanha and tartar emetic with the latter. It is also a tradition that the former are more suitable for subacute and chronic bronchitis, the latter for acute bronchitis. Senega, at least, appears to act exactly like ipecacuanha and tartar emetic, by irritation of the stomach. It has no known stimulant quality, and until it is shown to possess this it is misleading to call it a stimulant expectorant or to regard it as differing in respect of its expectorant action, pharmacologically or therapentically, from ipecacuanha. The expectorant action of squill is likewise due mainly to gastric irritation, though it may act on the centre as well. But the term "stimulant expectorant" was applied at a time when very little was known of the pharmacological action of expertorants. Seeing that, nearly a hundred years ago, Christison pointed out that "there is not, as some still imagine, any sound reason for avoiding it [squill] in acute affections of the chest on account of its supposed stimulant operation," the time would seem to be ripe for abandoning this division of expectorants into stimulants and depressants, as also for challenging the idea that ther $\theta$ is any known reason for avoiding squill or senega in acute bronchitis.

Where squill does definitely differ from these other three expectorants is that it has a digitalis-like action on the heart and is also a diuretic. It is especially in chronic bronchitis that these added actions would be likely to be of value. It is not improbable, therefore, that it acquired its reputation in chronic bronchitis for these added actions, which would be more likely to be displayed in the days when it was given in larger doses. But to regard it as having some kind of expectorant action different from ipecacuanha would seem to be misleading.

It would be beyond the limited scope of this paper to attempt to deal comprehensively with the many facts of the action of expectorants in regard to which our knowledge is deficient. As has already been hinted, this deficiency is, broadly speaking, of two kinds. One is the want of satisfactory explanation for the employment of a drug which has been handed down to us from an empiric age, and the other is incomplete knowledge of the physiological actions of the drugs.

An interesting example of the former is the use of hydrocyanic acid in cough. Hydrocyanic acid has been used for a long time as a common ingredient in cough mixtures, with the reputation that it "relieves an irritative cough." Its value for this purpose is usually scoffed at in modern textbooks of pharmacology, and the criticism is probahly well founded that the doses given are too small to have any direct effect, either on the cough centre or on the bronchial tubes. This, however, does not negative its employment, and a consideration of its pharmacological actions and the history of its therapeutic use will perhaps place it upon a more reasonable basis. It was originally given not by the mouth but as an inhalation, and in the British Pharmacopoeia of 1867 there was a "vapor acidi hydrocyanici" for the latter purpose. I believe that this practice originated in the use of inhalation of cherry-laurel water. As hydrocyanic acid is known to exert a local anaesthetic action which is especially marked on mucous membranes, there is at least the possibility that, when inhaled, it would exert this action on the ilpper air passages. Here again we seem to have no measurement of the dilution in which an effective sedative action of hydrocyanic acid would bo produced; but in any case the original use of hydrocyanic acid in cough was based upon a known pharmacological action. The practice of administering it by inhalation was given up probably from fear of accidents with so powerful a poison. But it still has a reputation for relieving cough when given by the mourh, and there is a pharmacological justification for its use even when given in this way. 
As has already been shown, cough and bronchial secretion can be excited by irritation of afferent branches of the vagus other than those of the respiratory mucous mem. brane, if this irritation is of sufficient intensity or duration, and the action of a whole group of expectorants is due to this. If a pharmacological irritation of the gastric mucous membrane can excite cough or bronchial secretion, it is difficult to see how a pathological irritation of the same surface can fail to do so likewise. Indeed, a " stomach cough," especially occurring in children, in whom these reflexes are more active, was well recognized by older physicians. The action of hydrocyanic acid may well be that, when given by mouth, it relieves cough by its anaesthetic action on the pharynx and stomach, checking, in fact, the very reflex which ipecacuanha excites. It could thus clear itself of a reputation for uncertainty, because, having no direct action on the respiratory system (in these doses), it would relieve cough only when this was secondary to pharyngeal or gastric irritation. Brunton, who, after preliminary scepticism, convinced himself of the reality of a "stomach cough," gave a similar explanation for the occasional relief of cough by sodium bicarbonate.

The action of hydrocyanic acid has been considered at some length because it warns against the condemnation of therapeutic experience merely because no obvious pharmacological explanation can be given; especially is this to be remembered when this scepticism regarding the usage of our forefathers is accompanied by an ignorance of their methods of administration.

The deficiency of our knowledge of expectorants, howerer, does not lie only in an occasional failure to give a reasonable explanation of therapeutic usage. We have much to learn, also, in regard to the physiological side, and an important example may be given. As is now well known, the vagus nerve supplies efferent branches not only to the bronchial mucous membrane, but also to the bronchial muscle. A drug like pilocarpine mar cause not only increased bronchial secretion, but also constriction of the bronchi. We seem to have no knowledge, however, as to whether, when bronchial servetion is provoked by reflex irritation-as, for example, by ipecacuanha-constriction of the bronchi is simultaneously produced. It may be that the secretory fibres of the vagus are more easily excited reflexly than the motor fibres. We have even less kiowledge of the peristaltic movement of bronchi or whether this is excited by expectorants. These problems would seem to be not insoluble by pharmacological methods. Christison has pointed out, in regard to ipecacuanha, that "expectoration is never so unequivocally augmented as when nausea is also excited." This has also been emphasized by others. It may be that this merely signifies that with larger doses of ipecacuanha there is a more copious bronchial secretion. It may be, on the other hand, that when closes large enough to produce nausea are given something else happens, and it is conceivable that this additional factor is a stimulation of the motor fibres of the vagus with a resulting peristaltic movement of the smaller bronchi. The conjecture is excusable in so far as it affords a basis for experiment.

\section{Concuuston.}

The object of this paper has been to attempt to give a clear exposition of the physiological basis for the action of expectorants and to summarize the present state of knowledge of secretory expectorants upon this basis. Suggestions have been made to reconcile the tenets of clinical experience with the known pharmacology of the drugs concerned, where this reconciliation seemed possible. In many respects our knowledge of the action of expectorants and of their relative values in different conditions is far from complete. Many of the points in dispute can be decided only by observation of the effects of expectorants in patients with respiratory disease, though the contribution which the pharmacological laboratory may yet make is not exhausted. Here, however, is a field of investigation for which ample clinical material is available. It is a field in which the general practitioner can play an important part.
Dr. H. Whitridge Davies (Leeds) considered that ammonium chloride acted only by its gastric irritant effect. When large quantities of this drug were taken in order to produce experimental acidosis the subject suffered from very considerable nausea. Moreover, Haldane had stated that in order to avoid emesis in experiments of this kind it was necessary to take it in very dilute solution. After absorption ammonium salts were converted into urea, and the amount which could be excreted into the bronchi must be infinitesimal.

Professor R. J. S. McDowall (London) said that it was well known by laryngologists that the bronchi contracted during inspiration. He thought that Professor Gunn's suggestion of combining the use of expectorants and adrenaline might well prove nost useful.

\section{DISCUSSION ON IMMUNITY."}

\section{I.-CERTAIN PRACTICAL ASPECTS OF IMMUNITY.}

R. A. O'BRIEN, M.D.,

Wellcome Physiological Research Laboratories, Beckenham, Kent.

Is reviewing the great amount of work being carried out in immunology and its vast literature, the general practitioner, medical or veterinary, has a right from time to time to ask of the workers in these fields what advances in medicine have resulted-advances so generally accepted as to be reasonably considered permanent, and which he must therefore be prepared to use in his practice. It was arranged that, as Professor Browning is dealing with the more attractive explanation of the phenomena underlying certain aspects of immunity, I should deal with what we may call the practical achievements of immunology in the recent past, and also survey briefly the tasks just ahead; since comparative medicine is represented at this Section, a review of these achievements in human and veterinary medicine side by side may not be out of place.

In endeavouring to estimate future tasks and possible lines of advance, what lessons can we learn from the immediate past? During the past century the death rate from many human diseases has been much reduced. Those that spring at once to the mind in this connexion are the intestinal diseases-typhoid, cholera, and drsentery, and typhus and plague. I do not know that we can draw from veterinary epidemiology a close parallel.

If we indeavour to draw up an order of merit for those who have achieved this reduction, we shall have to award the greater honour to the hygienist and sanitarian, who insists on general measures of common-sense cleanliness, fresh air, a decent allowance of space for the individualthat is, the avoidance of overcrowding-and the guarding of food and water supplies. Next in order would come the inmmunologist, who presents himself mostly with a syringe and needle. Last of all the clinician, when disease surmounts the barriers set up by the hygienist and vaccinator, to-day treats his patients with more knowledge than of old, and reduces the death rate either with "specific" remedies, such as antitoxin, as in diphtheria, or with greater clinical skill, until he to-day expects a mortality not more than half that of an earlier date.

But will the contribution of these three groups of medical workers bear the same relative proportion in the advances of the future? In considering these lines of advance the teachings of general epidemiology, and particularly those of experimental epidemiology, and of the influence of avitaminosis on susceptibility to infection, must be borne in mind. We are beginning to have a clearer idea of many points, thanks to the work of Topley and others, particularly of the exact way in which isolation of infected

* Report of a discussion at a joint meeting of the Sections of Pathology and Bacteriology and of Comparative Medicine at the Anqual Mrofessor T. J. Mackie was in the chair. 